\title{
Accuracy and Precision Analysis for a Biophotonic Assay of C-Reactive Protein
}

Philip James-Pemberton ${ }^{1,2}$, Urszula Łapińska ${ }^{1,2}$, Mark Helliwell ${ }^{1,2}$, Rouslan V. Olkhov ${ }^{1,2}$, Oliver J. Hedaux ${ }^{1,2}$, Christopher J. Hyde ${ }^{3}$ and Andrew M. Shaw ${ }^{1,2}$

\footnotetext{
${ }^{1}$ Biosciences, College of Life and Environmental Sciences, University of Exeter, UK

${ }^{2}$ Attomarker Ltd, Innovation Centre, Rennes Drive, Exeter, EX4 4RN, UK

${ }^{3}$ College of Medicine and Health, St Luke's Campus, Heavitree Road, Exeter EX1 2LU, UK
} 


\section{Abstract}

A multiplexed biophotonic assay platform has been developed using the localised particle plasmon in gold nanoparticles assembled in an array and functionalised for two assays: total IgG and C-reactive protein (CRP). A protein A/G (PAG) assay, calibrated with a NIST reference material, shows a maximum surface coverage of $\theta_{\max }=7.13 \pm 0.19 \mathrm{mRIU}$, equivalent to $1.5 \mathrm{ng} \mathrm{mm}^{-2}$ of $\mathrm{F}(\mathrm{ab})$-presenting antibody. The CRP capture antibody has an equivalent surface binding density of $\theta_{\max }=2.95 \pm 0.41 \mathrm{mRIU}$ indicating a $41 \%$ capture antibody availability. Free PAG binding to the functionalised anti-CRP surface shows that only $47 \pm 3 \%$ of CRP capture antibodies are correctly presenting Fab regions for antigen capture. The accuracy and precision of the CRP sensor assay was assessed with 54 blood samples containing spiked CRP in the range $2-160 \mathrm{mg} / \mathrm{L}$. The mean accuracy was $0.42 \mathrm{mg} / \mathrm{L}$ with Confidence Interval $(\mathrm{Cl})$ at $95 \%$ from -14.7 to $13.8 \mathrm{mg} / \mathrm{L}$ and the precision had a Coefficient of Variation (CV) of $10.6 \%$ with $95 \% \mathrm{Cl} 0.9 \%-20.2 \%$. These biophotonic platform performance metrics indicate a CRP assay with 2 - $160 \mathrm{mg} / \mathrm{L}$ dynamic range, performed in 8 minutes from $5 \mu \mathrm{L}$ of whole blood without sample preparation. 


\section{Introduction}

Novel biosensor events are often reported demonstrating a transduction of a detection event specific to a molecule of interest, usually under ideal conditions ${ }^{1-5}$. Less often, biosensors are put through an analysis of sensitivity and specificity to establish the accurate detection of the target molecule in biologically or environmentally relevant fluids ${ }^{6-8}$. Fewer biosensors still are then tested against other platforms and international standards to make the transition from technique to technology: no molecular biosensor based diagnostic devices received FDA 510(k) approval in the period 2018 to April $2019^{9}$. In part, the reason is because the clinically relevant accuracy and precision requirements demand technique fidelity that can be mass produced. The majority of the surviving technologies in the clinical chemistry laboratories in the EU are dominated by a few large-scale devices such as Roche Cobas, Abbott and Hitachi, all using a turbidimetric detection method. Curiously, circulating standards such as the RIQAS provided by Randox suggest there is significant variation in the accuracy of these techniques, presumably based on their chosen reference standards. The instruments and their underlying techniques are robust but require sample preparation such as clotting and consequently trained operators.

New techniques must also meet the precision and accuracy requirements to become clinically useful which places significant demands on the reproducible construction of sensor surfaces such as the orientation of antibodies ${ }^{10}$ and their durability over the cycles of precision and accuracy testing. Our biophotonic biosensor platform uses gold nanoparticles printed into a 150-element array as seeds, grown into larger scatter centres and functionalised with different antibodies to allow multiplexed analyte analysis, Figure 1. A two-step sandwich assay provides sensitivity and specificity to the target analyte and video capture of the array spot brightness changes produces an immuno-kinetic assay with results in 8 minutes. The conversion to array reading technique into a technology requires the sensor platform to be robust in complex, biologically relevant media ${ }^{11-18}$ with patient-topatient variability whilst preserving the accuracy and precision needed for regulatory approval and clinical diagnosis. 


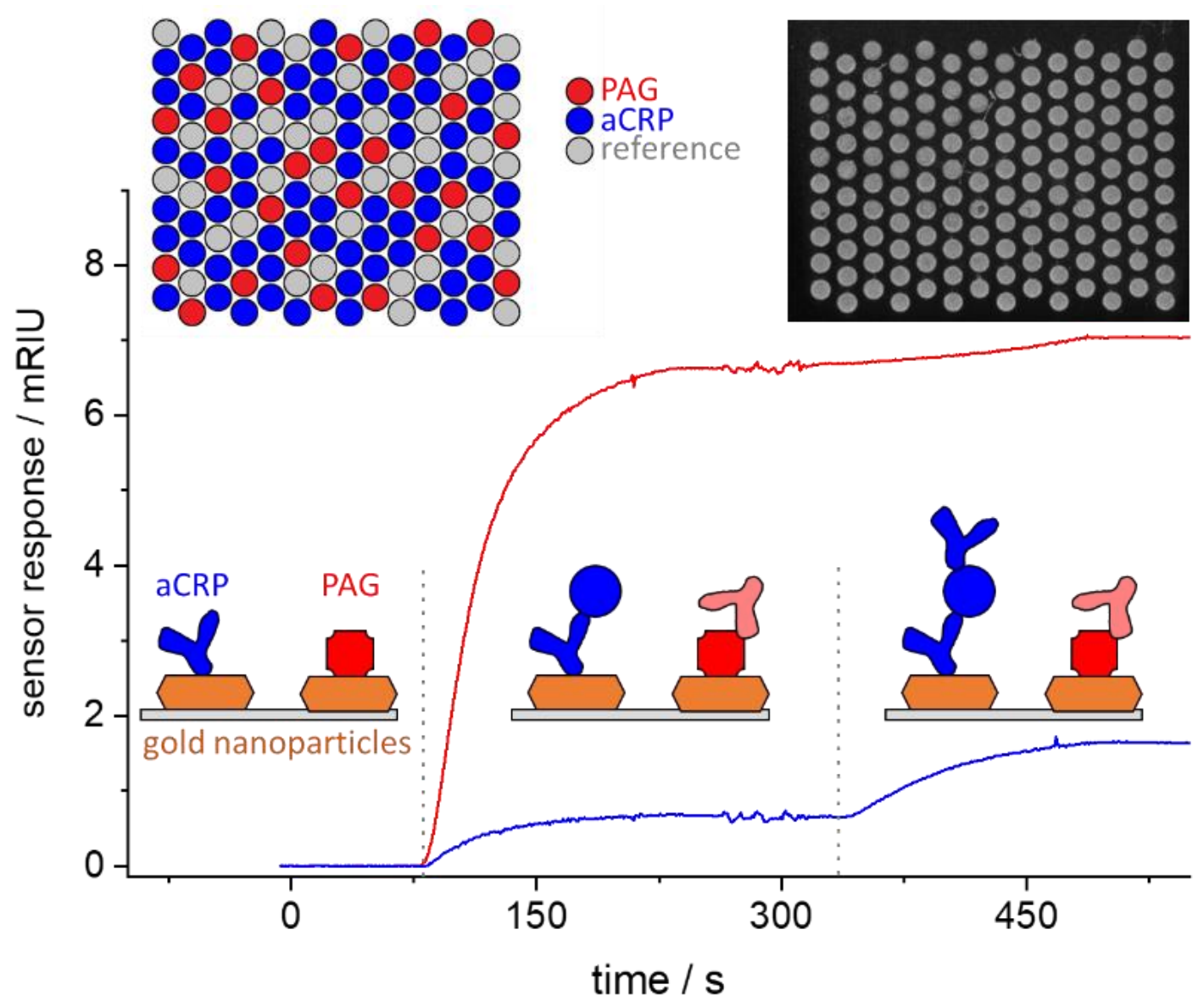

Figure 1 The biophotonic multiplexed array immuno-kinetic assay platform for total IgG and CRP analysis. There are three stages to the assay: capture of the analyte from the diluted bio-sample using PAG and aCRP; a wash step; and a detection step with a known concentration of aCRP, producing an evolving area-under-the curve for calibration.

Manufacture under regulatory control and subsequent production requires a set of quality control (QC) metrics defining the figures-of-merit of the assay that can be monitored during batch production. The assay platform has been optimised for two assays, C-reactive protein (CRP) and total IgG both have commercial interest. Total IgG provides useful information about antibody integrity ${ }^{19}$ and in-line $\mathrm{QC}$ of the materials on delivery supporting both the CRP assay and the total IgG assay. CRP is the target clinical assay and is the subject of $\sim 5,000$ publications per year (PubMed) as a sensitive but not specific biomarker of systemic inflammation in many conditions such as $\operatorname{sepsis}^{20}$. CRP has an important potential role in antibiotics stewardship providing a test before antibiotics are prescribed, despite its lack of specificity at low values is it useful in respiratory tract infections (RTI). ${ }^{21-23}$ Patients recruited to the RTI cohort by the GP with CRP less than $20 \mathrm{mg} / \mathrm{L}$ are likely to be self-resolving, 20-100 $\mathrm{mg} / \mathrm{L}$ watch and wait and at $100 \mathrm{mg} / \mathrm{L}$ or more are prescribed antibiotics ${ }^{24}$. Antibiotic 
stewardship with these guidelines reduces the use of antibiotics up to $35 \%$ and improves the diagnostics accuracy from $70 \%$ to $95 \%$. The use-case required clinically sets an accuracy standard.

Incorporating a CRP test into a care pathway and achieving clinical adoption requires regulatory approval: ISO 13485 leading to a CE mark in the EU or FDA approval in the US. Only the FDA sets explicit targets for the performance criteria of CRP assays based on the accuracy and precision over the specified range of intended use aligned with clinical utility

${ }^{25}$. Precision is well defined with intra-day and inter-day measures but accuracy is not: there is no internationally agreed CRP standard material adopted by all laboratories or manufacturers. The non-standardised accuracy implies that measurements in different hospitals are providing differences in accuracy as large as $20 \%$ which around a clinical decision point of $100 \mathrm{mg} / \mathrm{L}$ constitutes an error of $80 \mathrm{mg} / \mathrm{L}$ and $120 \mathrm{mg} / \mathrm{L}$ leading to regional differences in care pathway decisions. The RTI care pathway requires accuracy at each of the CRP cut-off levels which influence the clinical decision and would therefore expect a circulating standard and international convention.

Biosensors for CRP in primary or secondary care have a number of advantages: rapid delivery of the CRP test result; a large install base with a traceable accuracy standard; low blood sample volumes allow more frequent testing for trend analysis; and multiple testing provides a patient response profile rather than just a single measure - trend medicine. In this paper, we report the design and characterisation of a rapid CRP assay on a gold nanoparticle localised particle plasmon resonance array platform that address these advantages. The dynamic range and assay fabrication parameters are assessed along with the origins of the sensitivity. Finally, an accuracy and precision analysis was performed using a protocol, characterised against traceable reference materials, that is a potential first step in the evaluation of a technique on the journey to technology.

\section{Experimental Methods and Materials}

The CRP assay was performed on an in-house immuno-kinetic assay platform using sensing light scattering from an array of bio-functionalised gold nanoparticles; Light Scattering Array 
Reader (LiSCAR), a benchtop device described in detail elsewhere ${ }^{13-15,26-28}$. Briefly, ca. 3.5 $\mathrm{nm}$ seed gold nanoparticles are deposited with sciFLEXARRAYER S3 spotter (Scienion AG, Berlin) into an array of typically 150 elements. The arrays are removed from the printer and placed in a growth solution that develops the seed nanoparticles into truncated icosahedra with a diameter of approximately $60 \mathrm{~nm}$. The localised particle plasmon field has a penetration depth of $63 \mathrm{~nm}{ }^{16}$, consequently the light scattering properties are sensitive to the refractive index local to the nanoparticle surface. The nanoparticles are functionalised with a self-assembled monolayer ${ }^{13,28}$ to allow EDC-NHS coupling of biomolecules to the surface. The array is returned to the printer for functionalisation with the target assays: protein $A / G$ (PAG) for total IgG detection, anti-CRP for CRP detection and a set of control spots functionalised with BSA or FBR to correct for variations in temperature, non-specific binding and variations in the illumination field. The arrays are illuminated in total-internalreflection and imaged normal to the sensor surface with a video camera: the change in scattered light from the array elements is associated with increasing mass (local refractive index or relative permittivity) in the plasmon field penetration depth. The brightness change is calibrated for refractive index sensitivity by providing a switch of known refractive index, PBS and $2 \times$ PBS which corresponds to a refractive index change of $1.6 \mathrm{mRIU}$ (measured with Reichert AR200 digital refractometer): the kinetic data are presented as plots of mRIU vs time.

The immuno-kinetic assay is performed in three steps: (i) capture of the analyte from a diluted whole blood sample flowing over the surface; (ii) a running buffer wash step to remove the non-specifically bound material from the sensor surface; and (iii) an injection of detection antibody at fixed concentration, to complete a sandwich assay as shown in Figure 1. The evolution of the signal brightness is corrected for temperature and illumination field variations using the control spots and integrated over $120 \mathrm{~s}$ in the detection step to produce an Area-Under-the-Curve (AUC). AUCs are collected for standard samples to produce a calibration curve which is used to derive the final unknown (blinded) analyte concentration. The sensor surface can be regenerated with a low $\mathrm{pH}$ buffer and may perform in excess of 100 assays with limited degradation. Sets of 20 assays are profiled for mean variation in AUC for the CRP assay and show degradation of $5.8 \%$, stable over multiple repeats. The protein A/G surface density is calibrated using the NIST reference antibody (RM 8671) to produce calibrated binding site density ${ }^{29}$. 
Antibody binding on PAG via the Fc region presents the Fab region to the solution to bind the target analyte. EDC-NHS coupling of antibodies to the surface does not preserve orientation (or activity). However, the antibody orientation on the sensor surface can be interrogated using an Fc-presenting assay by passing PAG over the surface. The surface density and orientation of antibodies produce a measure of quality control in the manufacture of the assay arrays ${ }^{19}$.

The kinetic analysis of the assay response is performed using experimental data obtained from a range of low protein concentration samples to produce an accurate assessment of the Langmuir adsorption kinetic parameters derived from a simultaneous, global fit to the complete kinetic dataset ${ }^{17}$. The total bound protein surface density is characterised by $\theta_{\max }$ derived from the global fit - a measure of the total surface capacity which can be calibrated using a NIST standard material and compared with the theoretical predictions.

A reference CRP assay dataset was collected using the Alere Afinion CRP laboratory testing device following the recommended protocol.

\section{Materials}

The materials used in the course of the experiments were used without further purification, as certified by the suppliers. Sigma-Aldrich supplied phosphate buffered saline in tablet form (Sigma, P4417), phosphoric acid solution (85 \pm 1 wt. \% in water, batch W290017, Sigma 345245), Tween 20 (Sigma, P1379), N-(3-Dimethylaminopropyl)-N'-ethylcarbodiimide hydrochloride (commercial grade, E7750), N-hydroxysuccinimide $(98 \%, 130672$ ) and bovine serum albumin (>98\%, A3059). SAM molecules, linker $\mathrm{HS}-\mathrm{C}_{11}-\mathrm{EG}_{6}-\mathrm{OCH}_{2}-\mathrm{COOH}(>95 \%, \mathrm{TH}$ 003-M11.N6) and spacer HS- $\mathrm{C}_{11}-\mathrm{EG}_{3}-\mathrm{OH}$ (>95\%, TH 001-M11.N3), were supplied by ProChimia Surfaces. Blood media (whole blood, Na-citrate, EDTA and heparin tubes) were supplied by Cambridge Bioscience Ltd. Native human C-reactive protein $(>99 \%, 1 \mathrm{mg} / \mathrm{mL}$ and $0.09 \%$ sodium azide BioRad PHP277) and goat anti human CRP polyclonal antibody (BioRad 1707-0189G) were obtained from Bio-Rad. Recombinant PAG was supplied by ThermoFisher Scientific (21186). The assay running and dilution buffer was PBS with 0.005 $\mathrm{v} / \mathrm{v} \%$ Tween 20 and the regeneration buffer was $0.1 \mathrm{M}$ phosphoric acid solution in deionized water. Alere Afinion CRP tests were purchased from Abbott (1116522). A stock 
solution of $320 \mathrm{mg} / \mathrm{L}$ CRP was made by adding $48 \mu \mathrm{L}$ of Biorad solution to $102 \mu \mathrm{L}$ of blood. Subsequent lower concentrations of CRP were made by serial dilutions of the $320 \mathrm{mg} / \mathrm{L}$ stock blood solution with pure blood. The CRP concentration in the Cambridge Blood Media as supplied was measured as less than $5 \mathrm{mg} / \mathrm{L}$ using an Alere Afinion AS100 device.

\section{Results}

The initial set of experiments characterised the CRP capture antibody both in purity, surface binding density and orientation on tethering to the sensor surface. The protein binding density was evaluated with two assays: the surface antibody coverage calibrated with the NIST antibody standard and the antibody orientation with the PAG-Fc presenting assay. The NIST reference antibody binding to the printed PAG surface is shown in Figure 2(A) at three concentrations and fitted to the Langmuir adsorption isotherm equation simultaneously for all three concentrations to derive the kinetic parameters: maximum surface load $\theta_{\max }$, association rate constant $k_{\mathrm{a}}$ and dissociation rate constant $k_{\mathrm{d}}$, presented in Table 1 . The biophotonic CRP sensor was interrogated by first comparing the purity of the aCRP antibody material with the NIST antibody standard of known purity $96.6 \%{ }^{29}$ binding to the PAG. The observed aCRP load on PAG surface resulted in $\theta_{\max }=2.95 \pm 0.41$ mRIU, compared with the NIST antibody standard load characterised by $\theta_{\max }=7.35 \pm 0.09 \mathrm{mRIU}$.
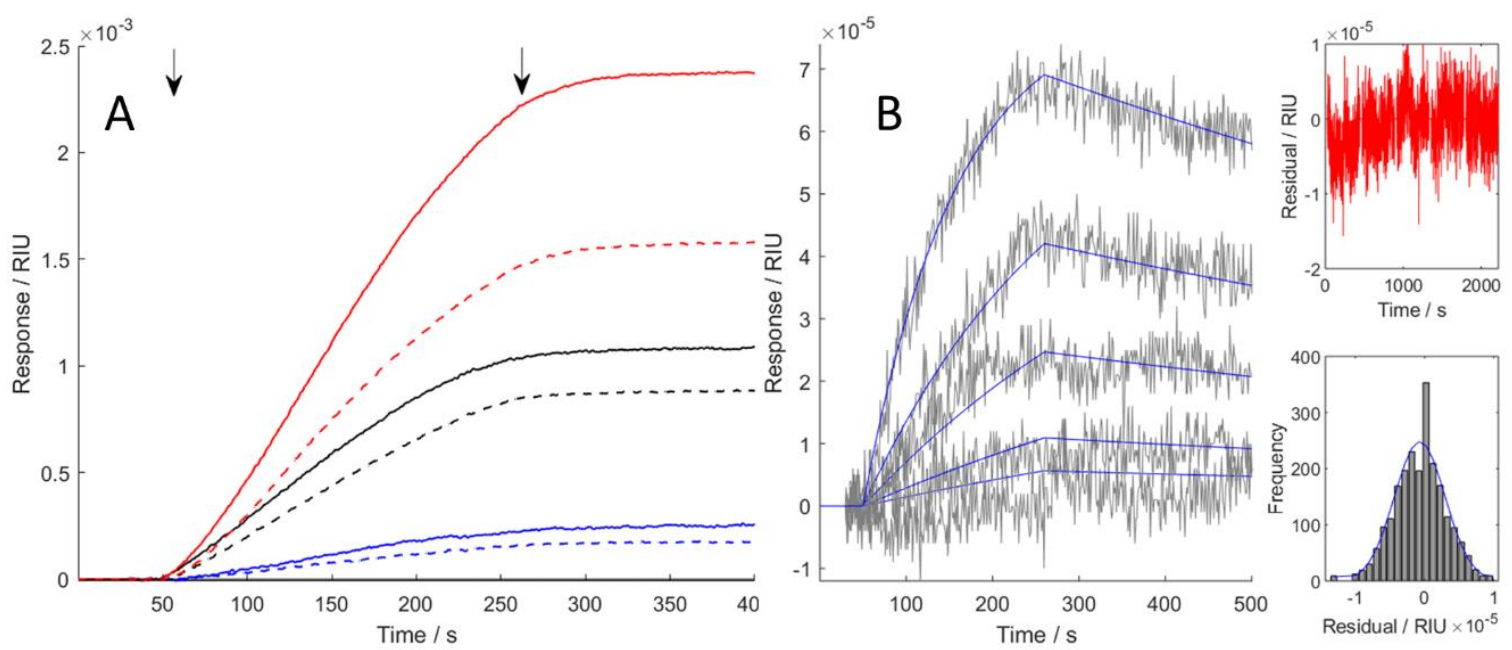

Figure 2 aCRP antibody and NIST antibody standard binding kinetics: (A) NIST standard material (solid lines) and aCRP antibody (dashed lines) binding to the PAG assay for $200 \mathrm{~s}$ followed by a running buffer wash using a flow rate of $6 \mathrm{~mL} / \mathrm{hr}$ (1, 5, 10 nM antibody samples in blue, black and red, from bottom to top); the arrows indicate the injection of aCRP and the onset of washing; B) PAG binding to the printed aCRP antibody assays a surface for available Fc binding sites $(1,2,5,10,25$ 
nM PAG concentration samples from bottom to top), with the global fit to the data and plots of the fit residuals and their distribution

The orientation of the aCRP printed to the CRP assay surface was determined using PAG in solution, flowing over five different concentration samples, Figure 2(B), from which the kinetic parameters were derived, Table 1.

Table 1 Binding Kinetic Parameters for PAG binding to Fc region on the CRP assay channel, CRP binding to the CRP assay Channel and aCRP binding to a PAG surface.

\begin{tabular}{ccccc}
\hline Sample & $\begin{array}{c}\text { NIST Binding to } \\
\text { PAG surface }\end{array}$ & $\begin{array}{c}\text { PAG-Fc Assay on } \\
\text { aCRP surface }\end{array}$ & $\begin{array}{c}\text { CRP Binding to } \\
\text { aCRP surface }\end{array}$ & $\begin{array}{c}\text { aCRP Binding to } \\
\text { PAG surface }\end{array}$ \\
\hline$\theta_{\max } / \mathrm{mRIU}$ & $7.35 \pm 0.09$ & $0.10 \pm 0.02$ & $0.17 \pm 0.01$ & $2.95 \pm 0.41$ \\
$k_{\mathrm{a}}\left(\mathrm{M}^{-1}-\mathrm{s}^{-1}\right) / 10^{5}$ & $2.14 \pm 0.64$ & $3.31 \pm 0.14$ & $23.0 \pm 3.6$ & $3.30 \pm 0.71$ \\
$k_{\mathrm{d}}\left(\mathrm{s}^{-1}\right) / 10^{-4}$ & $0.34 \pm 0.88$ & $7.72 \pm 0.45$ & $4.54 \pm 13$ & 0.1 (fixed) \\
$K_{\mathrm{D}} / \mathrm{nM}$ & $0.15 \pm 0.41$ & $2.34 \pm 0.24$ & $0.20 \pm 0.60$ & 0.03 \\
\hline
\end{tabular}

The quantitative CRP assay on the LiScAR platform is based on a calibration curve obtained by testing a set of standard samples over the dynamic range of the assay. The standards are prepared by spiking whole blood media with known concentration of CRP. The preparation volumetric accuracy was typically 5\%, principally from pipetting errors. The spiked blood sample is flowed over the sensor surface for $120 \mathrm{~s}$, the surface is then washed for $140 \mathrm{~s}$ and then the detection antibody at $100 \mathrm{nM}$ is flowed for $120 \mathrm{~s}$; integration over the detection step signal changes produces the AUC for the calibration curve in mRIU s. The same polyclonal antibody is used for both capture and detection. The AUC data obtained for CRP standard samples in the detection step, Figure $3 \mathrm{~A}$, were fitted to a five-parameter logistic calibration curve, Figure 3B. 

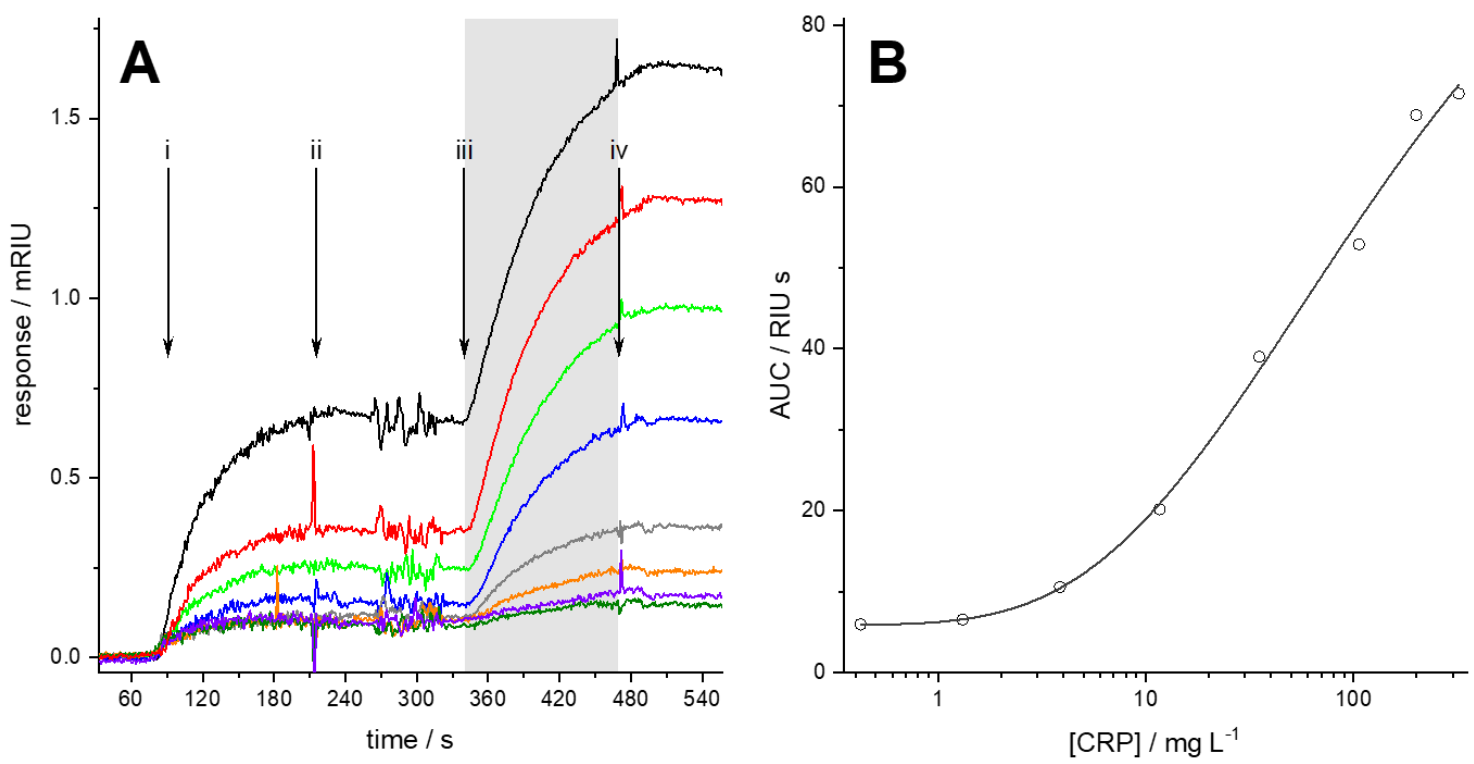

Figure 3 CRP Immunoassay: (A) kinetic data featuring sample CRP (spiked CRP concentrations in blood were 320, 200, 107, $36,12,3.9,0.44,1.3 \mathrm{mg} / \mathrm{L}$ from top to bottom) capture step from i to ii, a wash step of running buffer over the sensor from ii to iii, and subsequent binding of the detection a-CRP antibody from iii to iv - a 120s integration time; (B) fitting the integrated responses from the shaded area in panel A (circles) to a 5-parameter logistic function forms an assay calibration curve.

In general, the systematic nonspecific binding and the observed signal noise level result in a two standard deviation limit of detection (LOD) equivalent to $0.4 \mathrm{mg} / \mathrm{L}$ CRP concentration in the sample before the 100 -fold dilution employed in the assay protocol. The lower limit of quantification (LoQ), commonly assigned to five times limit of detection (LoD), is therefore 2 $\mathrm{mg} / \mathrm{L}$, and the upper quantification limit is estimated as $200 \mathrm{mg} / \mathrm{L}$ to be within $20 \%$ precision range of the calibration curves.

A set of spiked whole blood samples prepared for the accuracy and precision testing covered the range of CRP blood concentrations from 0.4 to $165 \mathrm{mg} / \mathrm{L}$ for the LiScAR. The sample set for the Alere Afinion was smaller, covering 5-150 mg/L CRP concentration range, appropriate for the measurement limits of the instrument. 

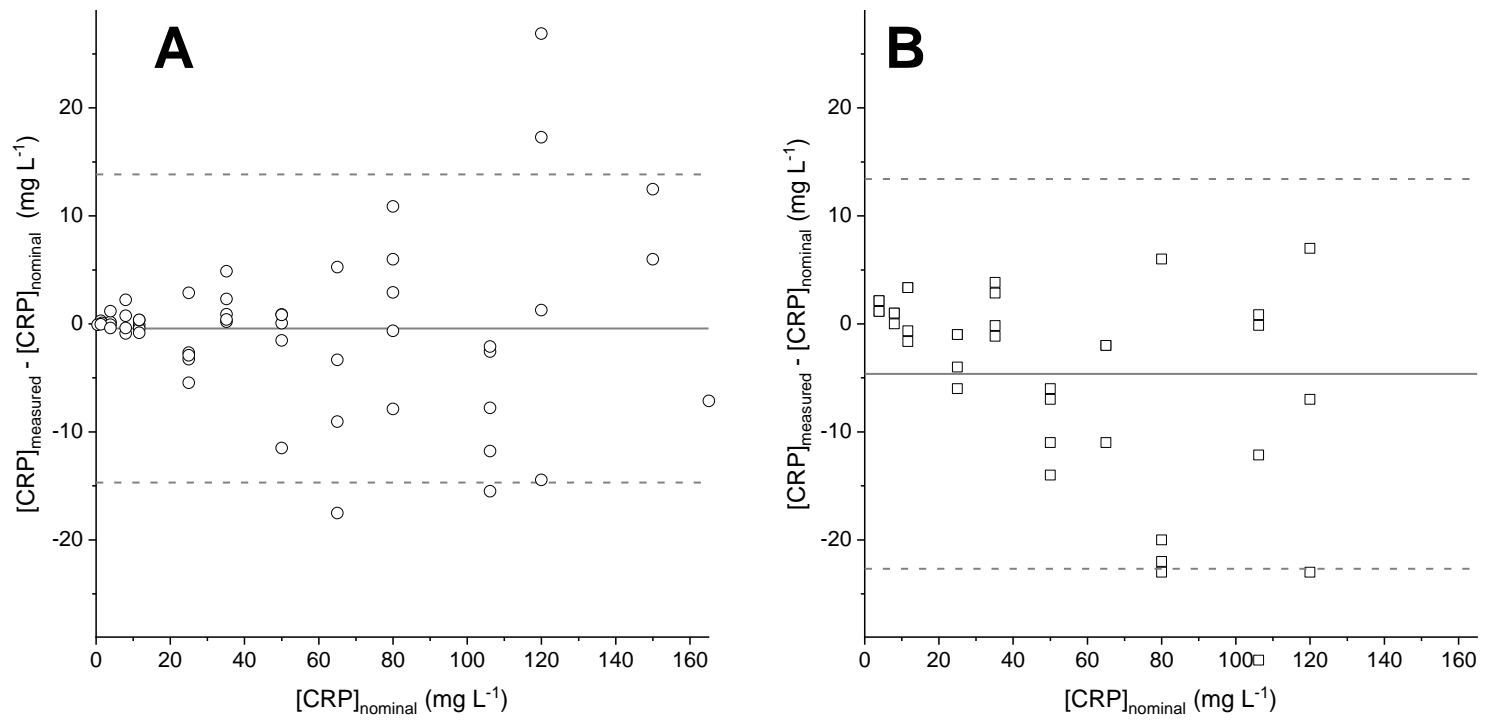

Figure 4 The difference plots for the CRP assay measurement and the nominal spiked CRP concentration in the whole blood media: (A) LiScAR (54 samples), and (B) Alere Afinion (37 samples) platforms; measured-nominal difference mean values are shown as solid lines with $95 \% \mathrm{Cl}$ as dashed lines

The assay accuracy is assessed by the differences between the nominal volumetric concentrations and the assay measured concentrations. The difference plot demonstrating 
the accuracy of the individual measurements for the LiScAR platform is shown in
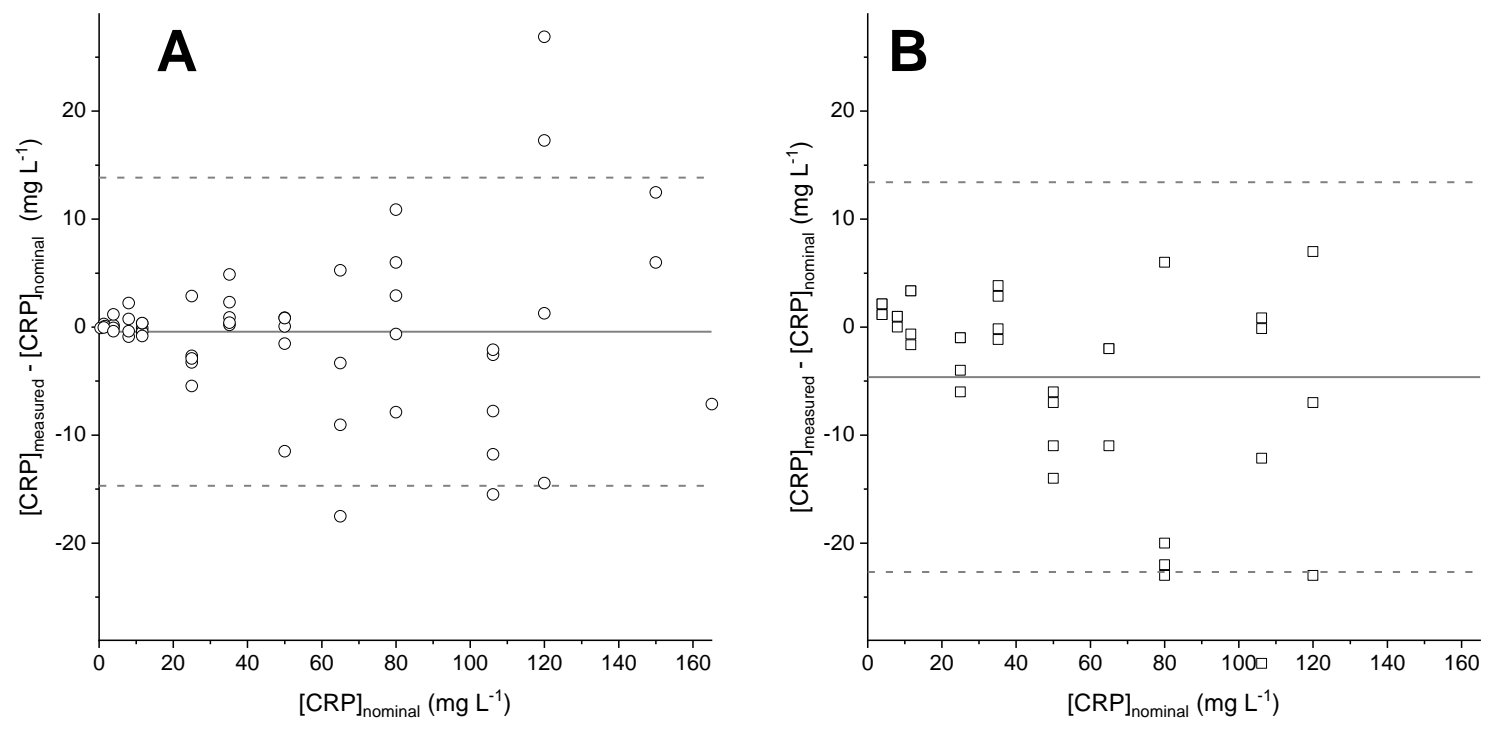

Figure 4A for the 54 tested blood samples, with a similar plot for the 37 measurements made with the Alere Afinion device in
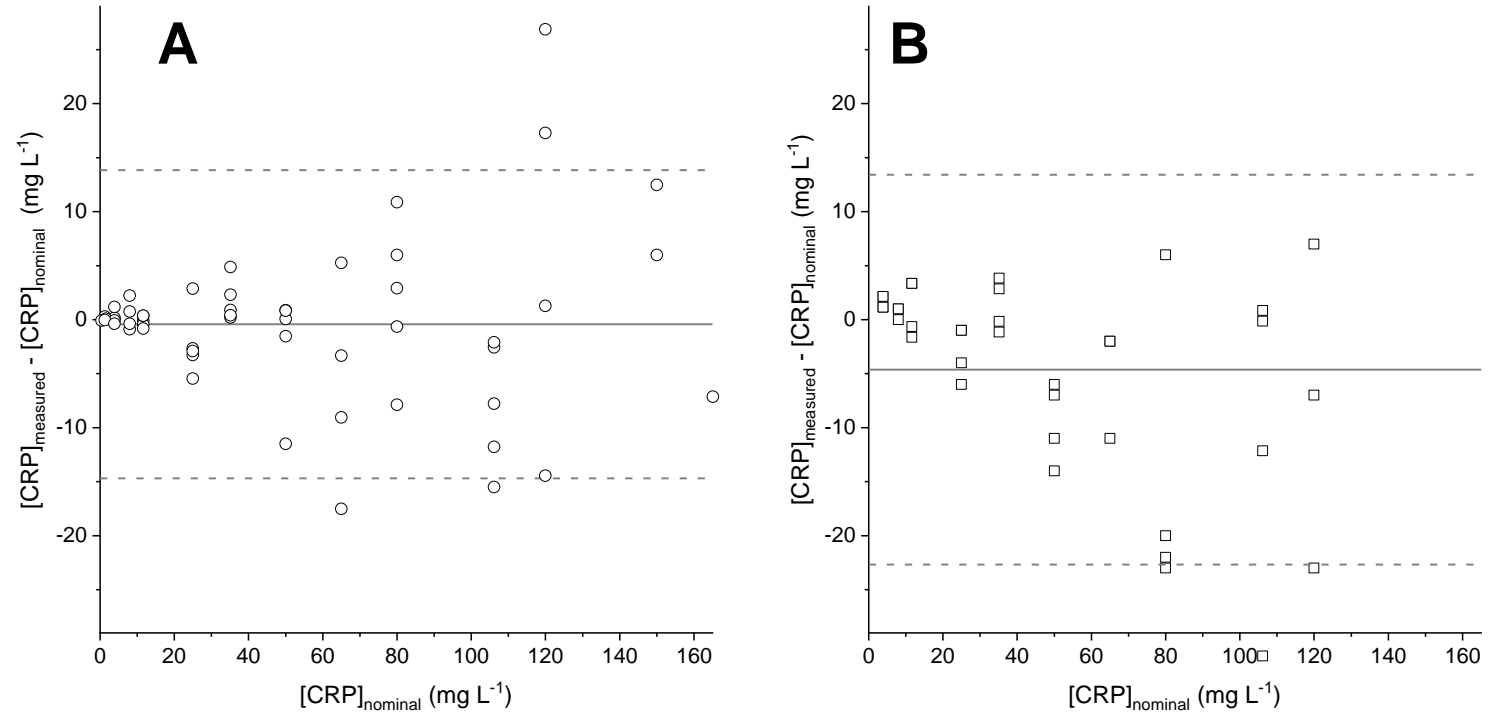
Figure 4B. The mean difference across the measured range of CRP concentrations for the LiScAR platform is $-0.42 \mathrm{mg} / \mathrm{L}$ with $95 \% \mathrm{Cl}$ from -14.7 to $13.8 \mathrm{mg} / \mathrm{L}$. The Alere Afinion measurements resulted in $-4.63 \mathrm{mg} / \mathrm{L}$ mean difference with $95 \% \mathrm{Cl}$ from -22.7 to $13.4 \mathrm{mg} / \mathrm{L}$. The negligible, $-0.42 \mathrm{mg} / \mathrm{L}$, mean measured-nominal [CRP] difference obtained for the LiScAR platform is expected since the instrument is calibrated using the same CRP stock material that was used in sample preparation. Correspondingly, a small bias of $-4.63 \mathrm{mg} / \mathrm{L}$ in Alere measurement results can be attributed to a number of effects: a systematic underreading of CRP concentrations; errors associated with the purity of the CRP sample and hence the accuracy of the nominal CRP concentration of the stock solution; and uncertainties in the Alere Afinion calibration. A systematic under-read is more plausible from looking at the data
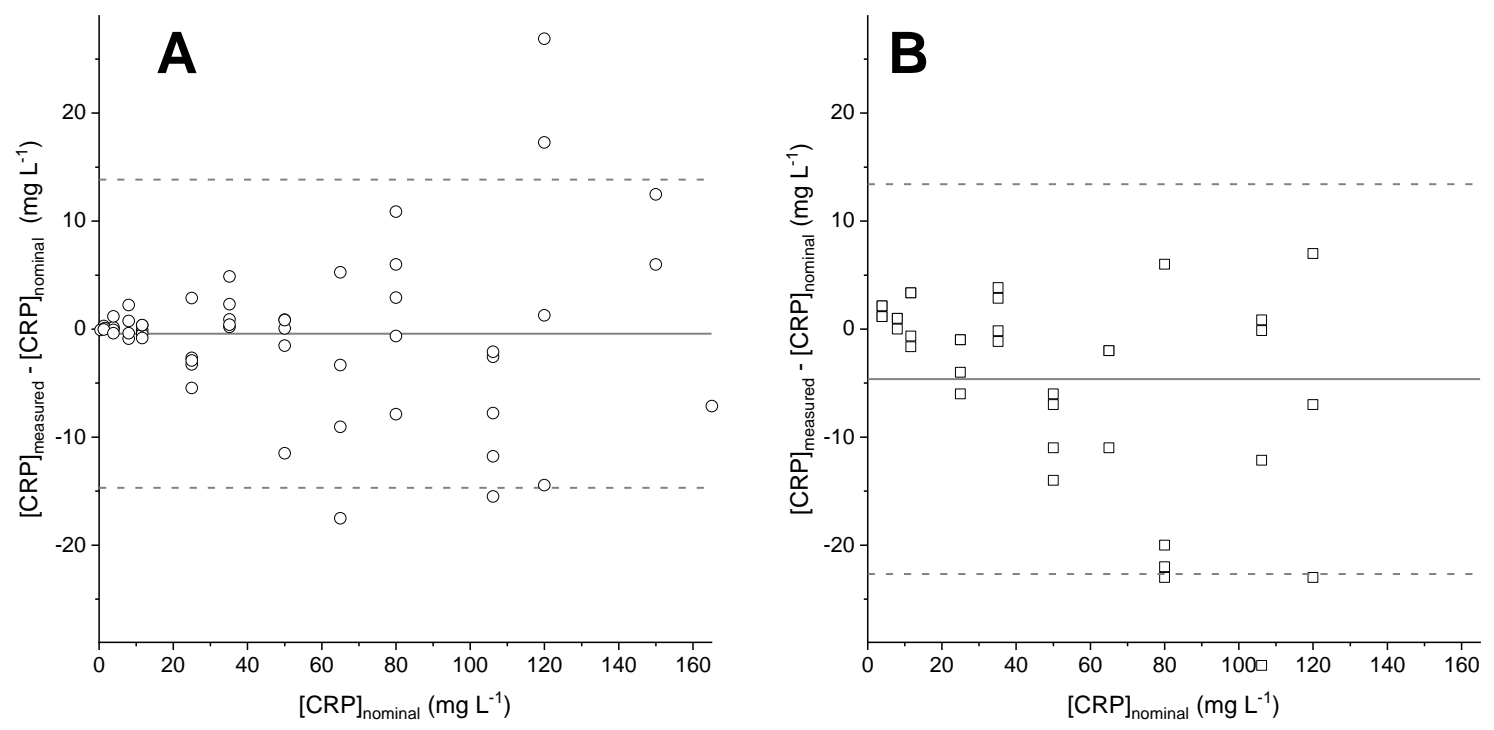
Figure 4B: there is a noticeable dependence of the Alere result on [CRP], and the bias would not be observed if the sample range was limited to about $5-40 \mathrm{mg} / \mathrm{L}$.
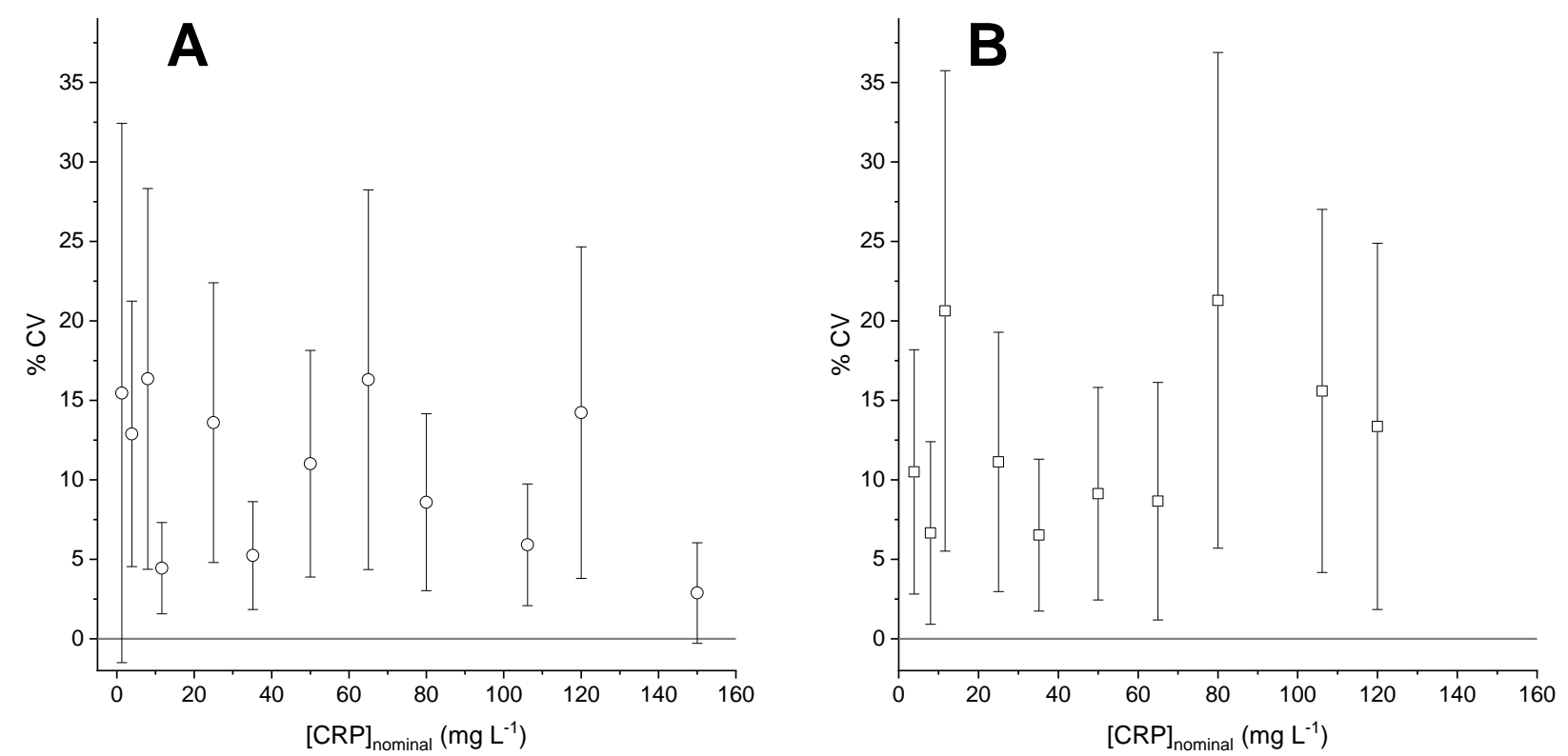

Figure 5 Coefficient of variation for the precision across the sample concentrations with a minimum of two repeated measurement from

The assay precision is characterised by Coefficient of Variation (CV) calculated as a standard deviation of the replicate at each concentration divided by the mean value for the repeated measurements and expressed as a percentage. The $95 \% \mathrm{Cl}$ for the $\mathrm{CV}$ are added to show the range expected with the current sample size, estimated using the method detailed ${ }^{30}$ elsewhere consider the uncertainty in the standard error in the standard deviation and the standard error in the mean for a small sample. Figure 5 shows the LiScAR and Alere Afinion precision data over the range of blood sample CRP concentrations. The average LiScAR assay precision has \%CV of $10.6 \%$ with $95 \%$ CI $0.9 \%-20.2 \%$ across the studied CRP concentration range. The analogous values for Alere Afinion are $\% \mathrm{CV}$ of 12.3 with $95 \% \mathrm{Cl}$ $2.0 \%-22.7 \%$.

\section{Discussion}

LiSCAR is a multiplexed platform that can perform 8 assays per 150 -spot array with up to 16 repeats of each assay. In addition there are 16 reference spots to eliminate non-specific binding, temperature dependence and illumination variations. The average array spot refractive index sensitivity is $3 \times 10^{-5}$ RIU which compares with standard continuous surface 
plasmon resonance sensitivities of $0.03 \times 10^{-5} \mathrm{RIU}$. The penetration depth of the plasmon field on the nanoparticle has however been recently measured as $c a .60 \mathrm{~nm}{ }^{16}$, indicating that the protein layer associated with the assay occupies a larger fraction of the plasmon field. Consequently, there is an intrinsic amplification or RI contrast ratio factor of 4.68 for the particles over the surface, reflected in the $\theta_{\max }{ }^{31}$. Fundamentally, plasmon sensors measure changes in local permittivity or refractive index which for protein sensors where the $\mathrm{RI}$ is constant, the biophotonics platforms are mass sensors. The mass of protein on the surface depends on the density of printed protein and the tethering efficiency which can be calibrated. We have chosen the NIST reference material to calibrate the total antibody load onto the PAG surface. The maximum surface coverage is given by $\theta_{\max }=7.35 \pm 0.09 \mathrm{mRIU}$ which may be compared with the random-close-packed antibody density. Assuming an antibody binding footprint of $91 \mathrm{~nm}^{2} \mathrm{RI}$ conversion factor of $1 \mathrm{ng} / \mathrm{mm}^{2} \sim 1 \mathrm{mRIU}{ }^{32}$ and plasmon field enhancement factor of $4.68^{31}$, gives a maximum close-packed antibody density on the surface of $12.8 \mathrm{mRIU}$, suggesting $56 \%$ maximum antibody coverage is achieved in the presented experiments. The NIST-PAG surface coverage provides a very simple QC measure for the quality of the printed surface.

For the CRP assay, the binding load of the aCRP polyclonal antibody is $\theta_{\max }=2.95 \pm 0.41$ mRIU, or $40 \%$ of NIST standard response, a variation that points to a sample integrity, purity and tethering efficiency. The latter may be due to fragmented antibodies binding to PAG: intact antibodies with full Fc biological activity, Fc-active fragments, either the Fc region completely fragmented from the Fab regions and so inactive biologically or Fc regions connected to one $F(a b)$ region retaining some activity ${ }^{19}$. There may also be a differential binding affinity for PAG depending on the species from which the antibody has derived. We have explored the NIST-PAG calibration elsewhere ${ }^{19}$ comparing the plasmonic purity with an LCMS analysis of 5 antibodies in fragmentation from different host animals showing limited affinity variability but supporting the fragmentation proposition. NIST is similarly critical as a reference material in these experiments surviving the mass spectrometry launch process intact. The NIST antibody standard has a quoted monomeric purity of $96.6 \%$ intact antibodies ${ }^{29}$ as confirmed by mass-spectrometric analysis. The $F(a b)$ biological integrity is reflected in binding capacity of the printed capture aCRP antibodies having a $\theta_{\max }=(0.17 \pm$ 0.01) $\mathrm{mRIU}$, correcting for the difference in mass of CRP and an antibody $\theta_{\text {max-Ab equivalent }}=$ 
$(0.22 \pm 0.01) \mathrm{mRIU}$, which in comparison with the NIST antibody standard load of $7.35 \mathrm{mRIU}$ suggests that only just over $3 \%$ of surface available for antibody tethering is occupied by biologically active CRP capture antibodies in the correct orientation.

The purity of the pAb capture antibody reducesthe sensor response by limiting the surface binding capacity of the CRP analyte and hence requires a complete set of QC parameters. The integrity of the surface can be interrogated by two QC assays: active Fab sites using the response to CRP and active Fc regions using the response to PAG. The average of $\theta_{\max }$ PAG binding aCRP surface is $0.10 \pm 0.02 \mathrm{mRIU}$ and for CRP binding to the same aCRP surface gives $0.17 \pm 0.01 \mathrm{mRIU}$, which correcting for the relative masses of PAG and CRP (50 kDa and $116 \mathrm{kDa}$ ) gives Fab $=49 \pm 11 \%$ and Fc $=51 \pm 11 \%$. Once formed however, the CRP assay surface is remarkably robust allowing in excess of 100 repeat analyses and leading to good accuracy and precision measurements. The average accuracy across the range CRP serum concentration range shows a mean difference of $-0.42 \mathrm{mg} / \mathrm{L}$ (95\% Cl -14.7 to $13.8 \mathrm{mg} / \mathrm{L}$ ) with precision of $10.6 \%$ (95\% $\mathrm{Cl} 0.9 \%-20.2 \%$ ) suggesting the current biophotonic platform has a clinically useful CRP range of $2-160 \mathrm{mg} / \mathrm{L}$ and an analysis time of eight minutes without any sample preparation.

The production properties of the assay are well defined in a set of QC parameters, such as surface protein loads and Fc presentation, suggesting that fabrication could be brought under process control with the correct supply of materials. The general FDA accuracy and precision requirements for a ligand-binding assay are met over the dynamic range of the CRP assay from the whole blood indicating that cells do not present a challenge for the surface. However, there are many remaining hurdles: matrix effects from different patients in particular with different lipids content, reliability of the sensor surfaces within day and between days, sensor shelf life, and the specificity of each assay in the multiplexed environment.

\section{Conclusions}

The current biophotonic multiplexed platform has demonstrated sufficient accuracy and precision required for a clinically useful CRP assay. The technique offers a clinically useful triage assay for CRP range of $2-160 \mathrm{mg} / \mathrm{L}$ with an average accuracy of $-0.42 \mathrm{mg} / \mathrm{L}(95 \% \mathrm{Cl}$ 14.7 to $13.8 \mathrm{mg} / \mathrm{L})$ and precision of precision of $10.6 \%$ (95\% Cl $0.9 \%-20.2 \%)$ from a $5 \mu \mathrm{L}$ of 
whole blood sample that requires no preparation and a result in 8 minutes. Critically, the integrity of the sensor surface can be interrogated with assays for surface protein density, Fc orientation and sample integrity parameterised against the NIST international standard material. These assay results provide a set of QC figures of merit that can easily be incorporated into in-line testing for batch production. It is estimated that up to $60 \%$ of blood tests are not present in time to assist in the diagnosis ${ }^{34}$ maybe we have a simple multiplex technology that can provide rapid triage diagnosis with clinical chemistry accuracy and precision.

\section{Declaration of Interests}

Prof Shaw is the Founder and Director of Attomarker Ltd which funded the research. With the exception of Prof Christopher Hyde who is an independent author, all of the authors are funded by Attomarker; Philip James-Pemberton has an industrial PhD sponsored by Attomarker Ltd.

\section{References}

1. D. Zhang and Q. Liu, Biosensors \& bioelectronics, 2016, 75, 273-284.

2. X. Huang, D. Xu, J. Chen, J. Liu, Y. Li, J. Song, X. Ma and J. Guo, The Analyst, 2018, 143, 5339-5351.

3. D. Xu, X. Huang, J. Guo and X. Ma, Biosensors \& bioelectronics, 2018, 110, 78-88.

4. $\quad$ L. J. Wang, Y. C. Chang, R. Sun and L. Li, Biosensors \& bioelectronics, 2017, 87, 686692.

5. M. B. Pepys and G. M. Hirschfield, The Journal of Clinical Investigation, 2003, 111, 1805-1812.

6. J. F. Masson, ACS sensors, 2017, 2, 16-30.

7. S. Mariani and M. Minunni, Anal Bioanal Chem, 2014, 406, 2303-2323.

8. J.-F. Masson, ACS sensors, 2017, 2, 16-30.

9. FDA, 510(k) Clearances, https://www.fda.gov/medical-devices/device-approvalsdenials-and-clearances/510k-clearances).

10. A. K. Trilling, J. Beekwilder and H. Zuilhof, The Analyst, 2013, 138, 1619-1627.

11. B. Jansen van Vuuren, T. Read, R. V. Olkhov and A. M. Shaw, Analytical Biochemistry, 2010, 405, 114-120.

12. R. V. Olkhov, J. D. Fowke and A. M. Shaw, Analytical Biochemistry, 2009, 385, 234241.

13. R. V. Olkhov, R. Parker and A. M. Shaw, Biosensors and Bioelectronics, 2012, 36, 1-5.

14. R. V. Olkhov and A. M. Shaw, Biosensors and Bioelectronics, 2008, 23, 1298-1302.

15. R. V. Olkhov and A. M. Shaw, Analytical Biochemistry, 2009, 396, 30-35.

16. T. Read, R. V. Olkhov and A. M. Shaw, Physical Chemistry Chemical Physics, 2013, 15, 6122-6127. 
17. T. Read, R. V. Olkhov, E. D. Williamson and A. M. Shaw, Analytical and Bioanalytical Chemistry, 2015, 407, 7349-7357.

18. A. M. Shaw, R. V. Olkhov, A. Jerdev and W. L. Barnes, in Nanoplasmonic Sensors, Springer, 2012, pp. 127-151.

19. P. P. Reader, R. V. Olkhov, S. Reeksting, A. Lubben, C. J. Hyde and A. M. Shaw, Anal Bioanal Chem, 2019, 411, 6487-6496.

20. M. Tan, Y. Lu, H. Jiang and L. Zhang, Journal of cellular biochemistry, 2019, 120, 58525859.

21. P. Azevedo, J. Costa and A. Vaz-Carneiro, Acta medica portuguesa, 2014, 27, 677680.

22. S. Searle-Barnes and P. Phillips, Journal of Paramedic Practice, 2017, 9, 27-32.

23. J. Cooke, C. Butler, R. Hopstaken, M. S. Dryden, C. McNulty, S. Hurding, M. Moore and D. M. Livermore, BMJ open respiratory research, 2015, 2, e000086.

24. M. F. Engel, F. P. Paling, A. I. Hoepelman, V. van der Meer and J. J. Oosterheert, Family practice, 2012, 29, 383-393.

25. FDA, Review Criteria for Assessment of C-Reactive Protein (CRP), High Sensitivity CReactive Protein (hsCRP) and Cardiac C-Reactive Protein (cCRP) Assays, FDA, 2005.

26. R. V. Olkhov, E. R. Kaminski and A. M. Shaw, Analytical and Bioanalytical Chemistry, 2012, 404, 2241-2247.

27. R. V. Olkhov and A. M. Shaw, Biosensors and Bioelectronics, 2008, 23, 1298-1302.

28. R. V. Olkhov and A. M. Shaw, Analytical biochemistry, 2010, 396, 30-35.

29. NIST, NISTmAb, Humanized IgG1k Monoclonal Antibody, https://wwws.nist.gov/srmors/certificates/8671.pdf, (accessed 03 07, 2019).

30. H. Abdi, Coefficient of variation, SAGE

Publications, Inc.; Thousand Oaks, CA, 2010.

31. B. J. van Vuuren, T. Read, R. V. Olkhov and A. M. Shaw, Anal Biochem, 2010, 405, 114-120.

32. J. S. Shumaker-Parry and C. T. Campbell, Analytical chemistry, 2004, 76, 907-917.

33. M. Baker, Nature, 2015, 521, 274-276.

34. J. Callen, A. Georgiou, J. Li and J. I. Westbrook, BMJ Quality \&amp;amp; Safety, 2011, 20, 194. 